\section{Preoperative CT scanning in chronic thromboembolic pulmonary hypertension}

Chronic thromboembolic pulmonary hypertension (CTEPH) occurs in around $4 \%$ of patients with pulmonary embolism and carries a poor prognosis. The problem is commonly treated with pulmonary thromboendarterectomy (PTE), which usually provides a permanent cure. Approximately $10 \%$ of patients die following this procedure, however, and at least as many show persistent pulmonary hypertension. Careful preoperative evaluation of CTEPH is essential, therefore, and pulmonary angiography is considered the most appropriate method of selecting patients for surgery. In an effort to find a less-invasive means of assessing these patients, a team from Germany have asked whether CT-scan findings can predict operability and surgical success.

Heinrich et al. retrospectively studied 60 patients who were treated with PTE for CTEPH. All patients had undergone a helical CT scan of the chest before surgery. By comparing preoperative and postoperative hemodynamic parameters with the CT findings, the investigators demonstrated that CT scanning is likely to be useful in predicting hemodynamic improvement after PTE. For example, a significant negative correlation was shown between postoperative pulmonary vascular resistance and the detection by CT scanning of central thrombi and dilated bronchial arteries. Patients without visible central thrombi on CT scans were more likely to show inadequate hemodynamic improvement after PTE.

Prospective studies done with new CT scan technology with improved resolution of the pulmonary vessels are now needed to validate these findings.

Original article Heinrich M et al. (2005) CT scan findings in chronic thromboembolic pulmonary hypertension: predictors of hemodynamic improvement after pulmonary thromboendarterectomy. Chest 127: 1606-1613

\section{Left-ventricular properties in diastolic heart failure}

Despite the fact that patients with diastolic heart failure usually have a normal ejection fraction, it has been proposed that significant abnormalities in left-ventricular systolic function are an important component of this condition. Baicu and colleagues hypothesized that the systolic properties of the left ventricle are, in fact, normal in these patients; the results of their study have recently been published in Circulation.

The researchers approached the problem by measuring multiple indices of left-ventricular systolic performance, function, and contractility in 75 patients with chronic diastolic heart failure and 75 controls without cardiovascular disease. Left-ventricular systolic performance was assessed by calculation of the stroke work generated by the ventricle. Left-ventricular systolic function, which relates left-ventricular performance to preload, was examined by measurement of preload-recruitable stroke work and ejection fraction. Finally, left-ventricular contractility was assessed by measuring the systolic stress-shortening relationship, endsystolic pressure-volume relationship, and peak positive $\mathrm{dP} / \mathrm{dt}$ (the first derivative of left-ventricular pressure versus time).

No significant differences in left-ventricular systolic performance, function or contractility were found between patients and controls, and so Baicu et al. conclude that these properties are unlikely to be linked to the pathophysiology of diastolic heart failure. They discuss these findings in the context of other work in the area, some of which has generated conflicting conclusions.

Original article Baicu CF et al. (2005) Left ventricular systolic performance, function, and contractility in patients with diastolic heart failure. Circulation 111:2306-2312

\section{Sildenafil for patients with pulmonary hypertension secondary to congestive heart failure}

Inhaled nitric oxide selectively decreases pulmonary vascular resistance in patients with pulmonary hypertension. This effect is shortlived, however, partly because of the hydrolysis of cGMP by the type 5 phosphodiesterase isoform (PDE5). The selective PDE5 inhibitor sildenafil-commonly used to treat erectile dysfunction-has been shown to enhance the vasodilatory effects of inhaled nitric oxide, and to block the rebound pulmonary vasoconstriction that follows treatment. In 